\title{
Translocation of active mitochondria during pig oocyte maturation, fertilization and early embryo development in vitro
}

\author{
Q. Y. Sun 1,3 , G. M. Wu², L. Lai ${ }^{2}$, K. W. Park², R. Cabot², \\ H. T. Cheong' ${ }^{2}$, B. N. Day ${ }^{2}$, R. S. Prather ${ }^{2}$ and H. Schatten ${ }^{1 *}$ \\ ${ }^{1}$ Department of Veterinary Pathobiology, University of Missouri-Columbia, Columbia, MO \\ 65211, USA; ${ }^{2}$ Department of Animal Science, University of Missouri-Columbia, Columbia, \\ MO 65211, USA; and ${ }^{3}$ State Key Laboratory of Reproductive Biology, Institute of Zoology, \\ Chinese Academy of Sciences, Beijing 100080, People's Republic of China
}

The distribution of active mitochondria during pig oocyte maturation, fertilization and early embryo development in vitro was revealed by using MitoTracker Green staining and confocal laser scanning microscopy. The regulation of mitochondrial translocation by microfilaments and microtubules was also studied. In oocytes collected from small follicles, strong staining of active mitochondria was observed in the cell cortex. Accumulation of active mitochondria in the peripheral cytoplasm and around the germinal vesicles was characteristic of fully grown oocytes collected from large follicles. Mitochondria accumulated in the perinuclear area during meiotic progression from germinal vesicle breakdown (GVBD) to anaphase I. Larger mitochondrial foci were formed and moved to the inner cytoplasm in mature oocytes. Compared with the oocytes matured in vivo, in which large mitochondrial foci were distributed throughout the cytoplasm, mitochondria were not observed in the central cytoplasm in most of the oocytes matured in vitro. Strong staining of mitochondria was observed in the first polar bodies in metaphase II oocytes.
In fertilized eggs, active mitochondria aggregated in the pronuclear region. Perinuclear clustering and a cortical ring were the most marked features of early cleavage. Active mitochondria were distributed in both inner cell mass cells and trophectoderm cells of the blastocysts. Disassembly of microtubules with nocodazole inhibited both mitochondrial aggregations to the germinal vesicle area and their inward movement to the inner cytoplasm during oocyte maturation, as well as the translocation of mitochondria to the peri-pronuclear region during fertilization, whereas disruption of microfilaments by cytochalasin B had no effects. These data indicate that: (i) oocyte maturation, fertilization and early embryo development in pigs are associated with changes in active mitochondrial distribution; (ii) mitochondrial translocation is mediated by microtubules, but not by microfilaments; and (iii) in vitro maturation conditions may cause incomplete movement of mitochondria to the inner cytoplasm and thus affect cytoplasmic maturation.

\section{Introduction}

Mammalian oocytes are arrested at the G2-M phase transition of the first meiotic division. In vitro, fully grown oocytes liberated from their follicles spontaneously reinitiate meiosis I, characterized by germinal vesicle breakdown (GVBD), chromatin condensation, spindle assembly, emission of the first polar body and progression to metaphase of the second meiotic division (MII), at which stage they undergo a second arrest until fertilization. After spermatozoa penetrate the oocyte, the second polar body extrudes, male and female pronuclei form and syngamy occurs to start early embryo development. Nuclear changes during oocyte maturation and fertilization are co-ordinated with movements of genetic material and organelles, and with biochemical changes in the cytoplasm to ensure

*Correspondence

Email: schattenh@missouri.edu normal embryo development. The normality of early embryogenesis is directly related to the ordered expression of these developmental programmes (Van Blerkom, 1991).

Of the numerous cytoplasmic changes that occur, the positioning of mitochondria may be involved in concentrating ATP or calcium to specific regions in oocytes or fertilized eggs to support normal developmental processes. Thus, the distribution of active mitochondria may be indicative of the energy or ion requirement of various key events during oocyte maturation, fertilization and early embryo development. In mice, the perinuclear accumulation of mitochondria between GVBD and metaphase I (MI) (Van Blerkom and Runner, 1984; Van Blerkom, 1991), and the polarized distribution of mitochondria to one half of the oocyte containing the MII spindle (Calarco, 1995) were observed and were regarded as one aspect of the developmental programme of cytoplasmic maturation. Previous observations also revealed that translocation of mitochondria is co-ordinated 
with changes in the location of microtubule-organizing centres and that the microtubule-mediated accumulation of mitochondria may be required for nuclear maturation of mouse oocytes (Van Blerkom, 1991). In hamster and human fertilized eggs, active mitochondria relocate to surround the pronuclei (Bavister and Squirrell, 2000; Van Blerkom et al., 2000). Changes in the location of active mitochondria are also suggested to correlate with the ability of hamster embryos to develop in vitro (Barnett et al., 1997; Bavister, 2000).

In pigs, the developmental ability of oocytes matured and fertilized in vitro is low compared with that of oocytes matured and fertilized in vivo, although progress with in vitro maturation and fertilization has been made (Prather and Day, 1998). Inadequate cytoplasmic maturation during in vitro maturation or abnormal in vitro fertilization is thought to impair embryonic development. The ultrastructure and distribution of mitochondria in pig oocytes have been observed by electron microscopy (Cran, 1985). However, unlike confocal microscopy, electron microscopy allows observations of only a limited number of samples. In pigs, the distribution of mitochondria during oocyte fertilization and early embryo development is largely unknown. In addition, the regulation of mitochondrial translocation in oocytes and fertilized eggs of this species has not been studied.

In the present study, MitoTracker Green staining was used in combination with confocal laser scanning microscopy to determine: (i) the distribution of active mitochondria during maturation of pig oocytes in vivo and in vitro; (ii) the mitochondrial distribution during fertilization and subsequent early embryo development in vitro; and (iii) the regulation of mitochondrial translocation by the microtubule and microfilament cytoskeleton.

\section{Materials and Methods}

In vitro maturation and fertilization of oocytes and in vitro culture of early embryos

In vitro maturation of pig oocytes was conducted in a chemically defined medium (Abeydeera et al., 1998). Briefly, oocytes were aspirated from antral follicles of ovaries collected from slaughtered prepubertal gilts. Oocytes from large follicles (3-6 $\mathrm{mm}$ in diameter) were assumed to be meiotically competent and were used for maturation culture. Oocytes from small follicles (0.5-2.0 $\mathrm{mm}$ in diameter) were also collected for mitochondrial evaluation. After washing three times with Hepes-buffered Tyrodes's lactate containing $0.1 \%(\mathrm{w} / \mathrm{v})$ polyvinyl alcohol, each group of 50 cumulus-enclosed oocytes was cultured for $44 \mathrm{~h}$ at $39^{\circ} \mathrm{C}$ in an atmosphere of $5 \% \mathrm{CO}_{2}$ in air in a 500 $\mu$ ldrop of tissue culture medium (TCM)-199 supplemented

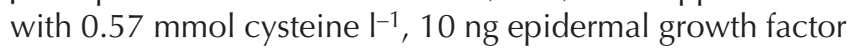
$\mathrm{ml}^{-1}$ (Sigma Chemical Co., St Louis, MO), 10 iu FSH ml-1 (Sigma), 10 iu hCG ml-1 (Sigma) and 0.1\% (w/v) polyvinyl alcohol.
IVF was carried out as reported by Han et al. (1999), with only minor modifications. Oocytes were inseminated in a $100 \mu \mathrm{l}$ drop of modified Tris-buffered medium containing $0.2 \%(\mathrm{w} / \mathrm{v})$ BSA and $2 \mathrm{mmol}$ caffeine $\mathrm{I}^{-1}$ with frozenthawed spermatozoa $\left(5 \times 10^{5}\right.$ cells $\left.\mathrm{ml}^{-1}\right)$. Six hours after insemination, oocytes were removed from the fertilization medium and cultured for up to 6 days in $500 \mu \mathrm{l}$ North Carolina State University (NCSU)-23 medium (Petters and Wells, 1993) containing 4 mg BSA ml-1.

\section{Collection of oocytes matured in vivo}

Gilts were observed for spontaneous oestrus. Between 42 and $48 \mathrm{~h}$ after oestrus was detected, two gilts were anaesthetized for surgical recovery of in vivo matured oocytes. Ovulated oocytes were collected by flushing the oviduct with Hepes-buffered Tyrodes's lactate containing $0.1 \%(\mathrm{w} / \mathrm{v})$ polyvinyl alcohol. Cumulus cells attached to the oocytes were removed by treatment with $0.3 \%(\mathrm{w} / \mathrm{v})$ hyaluronidase in Hepes-buffered Tyrodes's lactate containing $0.1 \%(\mathrm{w} / \mathrm{v})$ polyvinyl alcohol.

\section{Treatments}

Oocytes were cultured in maturation medium with or without the microtubule assembly inhibitor nocodazole $\left(100 \mu \mathrm{mol} \mathrm{I}^{-1}\right)$ or the microfilament organization inhibitor cytochalasin B (500 nmol $\mathrm{I}^{-1}$ ) for $44 \mathrm{~h}$ to evaluate the regulation of mitochondrial translocation by the cytoskeleton during oocyte maturation.

Oocytes matured in vitro for $44 \mathrm{~h}$ were fertilized in vitro for $6 \mathrm{~h}$ and transferred to the NCSU medium containing either $500 \mathrm{nmol}$ cytochalasin $\mathrm{B} \mathrm{I}^{-1}, 100 \mu \mathrm{mol}$ nocodazole $\mathrm{I}^{-1}$ or $1 \mu \mathrm{mol}$ taxol $\mathrm{I}^{-1}$ and subsequently cultured for up to 24 $\mathrm{h}$ to investigate the effects of cytoskeletal modulators on mitochondrial migration during fertilization.

\section{Confocal microscopy of active mitochondria}

A stock solution of MitoTracker Green FM at a concentration of $1 \mathrm{mmol} \mathrm{I}^{-1}$ was prepared in dimethyl sulfoxide (DMSO) and stored at $-20^{\circ} \mathrm{C}$. Oocytes, zygotes and early embryos were stained for active mitochondria in maturation medium or NCSU medium containing $0.5 \mu \mathrm{mol}$ cell permeant MitoTracker Green ${ }^{-1}$ (Molecular Probes, Eugene, OR) for $30 \mathrm{~min}$ at $39^{\circ} \mathrm{C}$ in $5 \% \mathrm{CO}_{2}$ in air. After washing three times, each for $20 \mathrm{~min}$ at $39^{\circ} \mathrm{C}$ in PBS, tissues were fixed in $2 \%(\mathrm{w} / \mathrm{v})$ paraformaldehyde in PBS for $1 \mathrm{~h}$ at room temperature. Oocytes, zygotes and early embryos were washed twice for 10 min each in PBS containing $0.3 \%$ (w/v) PVA (Sigma) and 1\% (v/v) Triton-X100 (Sigma) and stained with $10 \mu \mathrm{g}$ propidium iodide $\mathrm{ml}^{-1}$ for $10 \mathrm{~min}$ to detect DNA. After washing twice in PBS, the oocytes, zygotes and embryos were mounted on glass slides and observed using laser scanning confocal microscopy (BioRad, Hercules, CA). For observation of MitoTracker staining, the $488 \mathrm{nM}$ line and QH515/30 emission filter were used. The $568 \mathrm{nM}$ line was used to excite propidium 
iodide and emitted light was observed by using a HQ600/40 emission filter to visualize DNA. Data were saved to a disk and processed using Photoshop 5.5 software. At least 30 samples in each group were analysed in three repeated experiments except for the control group of in vivo matured oocytes $(n=17)$.

\section{Results}

\section{Distribution of active mitochondria during pig oocyte maturation}

Aggregation of active mitochondria in the cortex was a common feature of oocytes at the GV stage. In GV stage oocytes derived from small follicles of $0.5-2.0 \mathrm{~mm}$ in diameter, strong staining of a peripheral active mitochondrial band was observed in approximately half (19/40) of the oocytes (Fig. 1a). In the other oocytes (21/40), in addition to the mitochondrial aggregation in the cortex, some mitochondria were distributed in the sub-cortical region, but few were localized around the germinal vesicles or in the centre of the cytoplasm (Fig. 1b). The mitochondria were distributed homogeneously in the cell cortex (Fig. 1c). Sub-plasma membrane distribution of mitochondria was also a characteristic of GV stage oocytes derived from larger follicles of 3-6 mm in diameter; however, mitochondria were dispersed to the inner cytoplasm to different degrees in different oocytes (Fig. 1d-f), and in particular, mitochondria were mostly accumulated in the perinuclear region in many oocytes (15/31) (Fig. 1e,f). In all 37 oocytes that reached metaphase I or anaphase I after 24 or $30 \mathrm{~h}$ of culture, mitochondria were aggregated in the perinuclear area (Fig. $1 \mathrm{~g})$. The major characteristics of mitochondrial distribution in MII oocytes matured in vitro were as follows: (i) in most of the matured oocytes $(61 / 68)$, mitochondria were located more centrally, although few, if any, translocated to the very centre of the oocytes (Fig. 1h); (ii) relatively few but larger mitochondrial foci were distributed evenly in the cytoplasm except for the central region in all mature oocytes observed (Fig. 1h,i); (iii) strong staining in the sub-plasma membrane region was no longer apparent (Fig. $1 \mathrm{~h}, \mathrm{i}$ ); and (iv) strong active mitochondrial staining was common in the first polar bodies (51/68) (Fig. 1h,i). In all seven oocytes derived from large follicles that failed to undergo GVBD after $44 \mathrm{~h}$ of culture, mitochondria were located both around the GV and close to the plasma membrane, and no large mitochondrial foci were observed (data not shown).

Compared with the oocytes matured in vitro, the most marked feature of oocytes matured in vivo was that mitochondria were distributed throughout the cytoplasm, although mitochondria were more abundant in the peripheral cytoplasm than in the inner cytoplasm in most of the oocytes. Of the 17 samples observed, mitochondrial foci filled the entire ooplasm in 12 oocytes (Fig. 1j), but were absent in the central cytoplasm in three oocytes (Fig. $1 \mathrm{k}$ ) and were distributed in the central cytoplasm in two oocytes (Fig. 1l). Strong staining was also observed in the first polar body (Fig. 1I).

\section{Distribution of active mitochondria in fertilized eggs}

As expected, the tails, particularly the principal segments, of spermatozoa attached to the surface of the zonae pellucidae of fertilized eggs were stained by MitoTracker Green (Fig. 2a). After fertilization, mitochondrial aggregations were associated with the pronucleus (Fig. 2b), close to the plasma membrane (Fig. 2b,c), or between the pronucleus and the plasma membrane (Fig. 2c) in all 37 fertilized eggs examined, although some mitochondrial foci were observed in the inner cytoplasm of some fertilized eggs (Fig. 2b,d). Mitochondria were associated with both pronuclei (Fig. 2e). There was typically strong mitochondrial staining in the second polar bodies (21/37) $24 \mathrm{~h}$ after insemination (data not shown).

\section{Distribution of active mitochondria during early embryo development in vitro}

During early cleavage, mitochondria aggregated in the region in which the blastomeres were still in contact (Fig. 2f). The consistency of perinuclear clustering and of the cortical ring of mitochondria in the blastomeres was another characteristic feature (Fig. 2g). In morula, mitochondria were distributed mainly in the outer region of the blastomeres (data not shown). Active mitochondria were distributed homogeneously in both inner cell mass cells and trophectoderm cells in all pig blastocysts (Fig. 2h,i).

\section{Regulation of mitochondrial translocation by microtubule and microfilament modulators during oocyte maturation and fertilization}

When oocytes at the GV stage were cultured for $44 \mathrm{~h}$ in medium containing $500 \mathrm{nmol}$ cytochalasin $\mathrm{B} \mathrm{I}^{-1}$, meiotic progression was arrested at MI. Mitochondria migrated to the inner cytoplasm in the control MIl oocytes (29/36) (Fig. 3a), whereas mitochondria aggregated in the region near to the $\mathrm{Ml}$ chromosomes in most cytochalasin B-treated oocytes (34/40) (Fig. 3b,c), although a few mitochondrial foci were observed in the inner cytoplasm (Fig. 3c). In all five cytochalasin Btreated oocytes that failed to undergo GVBD, there were numerous mitochondria aggregated around the GV (Fig. 3d). In oocytes treated for $44 \mathrm{~h}$ with $100 \mu \mathrm{mol}$ nocodazole $\mathrm{I}^{-1}$, MI chromosomes formed, but further progression of meiosis was inhibited. Inward and perinuclear accumulation of mitochondria were blocked in all oocytes $(n=36)$. Mitochondria were aggregated mainly in the peripheral cytoplasm (Fig. 3e), although in some cases (15/36) a few mitochondria were distributed around the $\mathrm{Ml}$ chromosomes (Fig. 3f).

Microfilament disruption did not influence the aggregation of mitochondria around the pronuclear region in all 36 fertilized eggs examined at $24 \mathrm{~h}$ after insemination (Fig. 3g). When the two pronuclei were closely apposed, mitochondria were aggregated either around or between the two pronuclei. In contrast, the microtubule disruptor nocodazole inhibited aggregation of mitochondria in the 

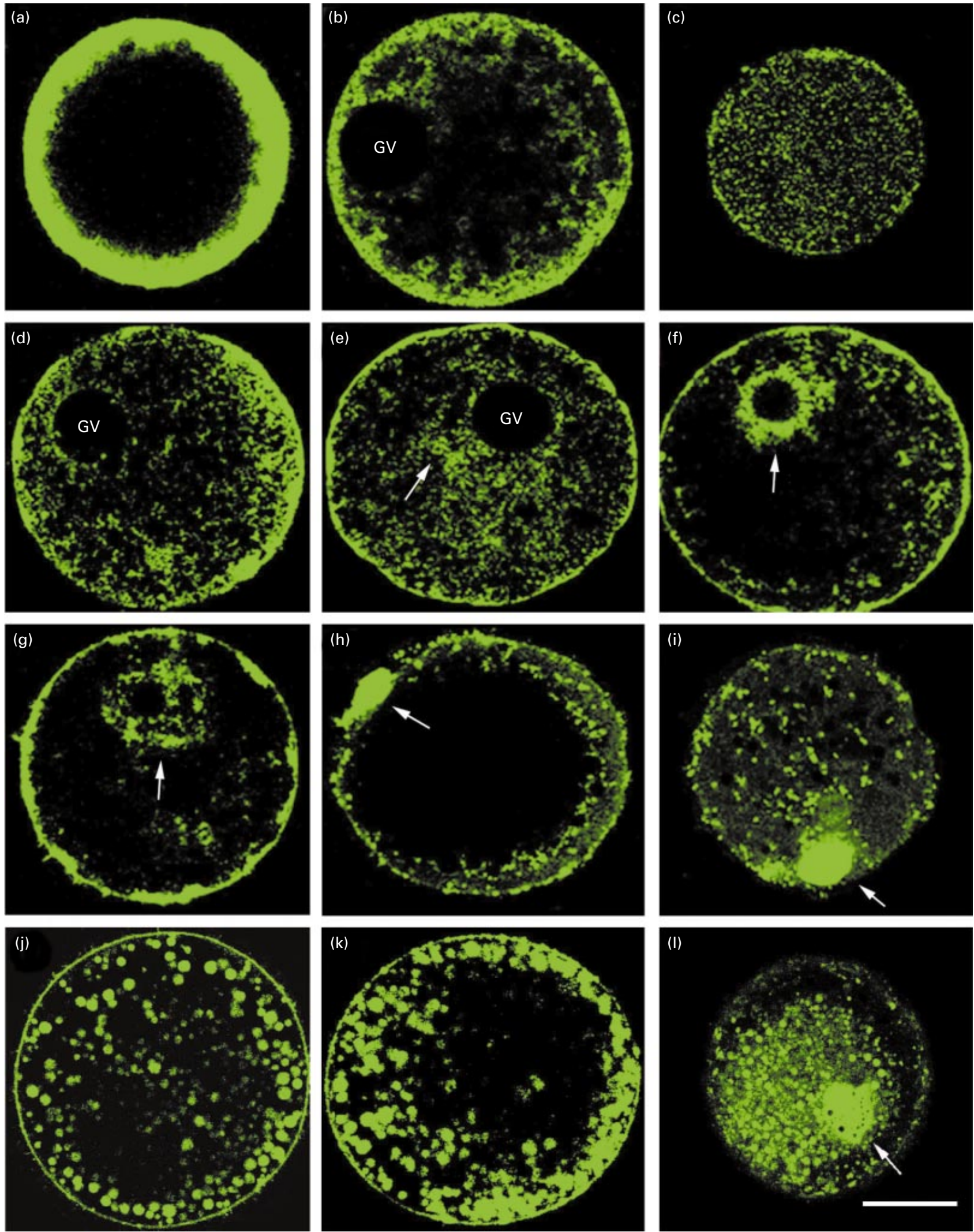

Fig. 1. Distribution of mitochondria during maturation of pig oocytes. (a) An example of a peripheral band of mitochondria in a germinal vesicle (GV) oocyte derived from a small follicle. (b) A GV oocyte from a small follicle, indicating the distribution of mitochondria in the sub-cortical region, but few distributed in the vicinity of GV. (c) Mitochondria were distributed homogeneously in 
peri-pronuclear region in 30 of 41 eggs examined. Mitochondrial foci were no longer observed and mitochondria were distributed homogeneously throughout the cytoplasm (Fig. 3h). Although taxol inhibited the centration of pronuclei, it did not affect aggregation of mitochondria in the vicinity of the pronuclei (28/35) at $24 \mathrm{~h}$ after insemination (Fig. 3i).

\section{Discussion}

Mitochondrial ultrastructure and changes in distribution during maturation of pig oocytes have been studied by electron microscopy. These studies revealed that mitochondria are clustered at the periphery of the cell before injection of human chorionic gonadotrophin (hCG), but disperse during maturation (Cran, 1985). In the present study, MitoTracker staining revealed a similar pattern of mitochondrial distribution. The restricted peripheral distribution of mitochondria was also reported in sheep and bovine oocytes (Cran et al., 1980; Sun and Qin, 1989). This pattern of distribution may be related to the high energy requirement in the cortex, as oocytes require cumulus cell support at this stage, and intimate association between the oocyte and the cumulus cells is maintained by gap junctions from cumulus cell process endings on the surface of the oolemma (Fleming and Saacke, 1972; Eppig, 1982). In addition to the sub-membrane aggregation, mitochondria accumulated progressively to the GV area before culture in immature oocytes collected from large follicles. Van Blerkom (1991) also observed the gradual perinuclear accumulation of mitochondria before GVBD in mouse oocytes. The accumulation of active mitochondria in the perinuclear region may not be related to the synthetic activity of the nucleus, as developing oocytes characteristically have high RNA synthesis, but fully grown oocytes cease rRNA synthesis, and no RNA synthesis is observed after initiation of meiosis (Prather, 1993; Sun and Qin, 1990; Sun et al., 1996). It is possible that perinuclear accumulation of mitochondria plays a role in GVBD. One possibility is that the specific patterns of perinuclear mitochondrial aggregation provide ATP for GVBD. Studies on mitochondrial distribution and ATP content in human pronuclear embryos show that a decrease in the number of mitochondria is correlated with a diminished capacity for ATP generation (Van Blerkom et al., 2000). However, other studies indicate that meiotic maturation occurs in both mouse and human oocytes over a wide range of ATP concentrations (Van Blerkom et al., 1995) and that mitochondrial contribution to ATP concentrations may be small in early development (Trimarchi et al., 2000). Furthermore, ATP production was reported not to be the mechanism whereby glucose mediates a stimulatory action on meiosis in mouse oocytes (Downs and Mastropolo, 1994). Another possibility is that perinuclear mitochondrial clustering plays a role in GVBD by modulating local free calcium concentrations, as increases in endogenously derived intracellular calcium are a prerequisite for GVBD in pig oocytes (Kaufman and Homa, 1993) and mitochondrial distribution is related to the ability to regulate intracellular homeostasis, as revealed in hamster embryos (Lane and Bavister, 1998). Mitochondrial aggregation around the GV was also observed in oocytes arrested at the GV stage after $44 \mathrm{~h}$ of culture, indicating that aggregation of active mitochondria in the GV area is not sufficient to induce GVBD, although this may be one of the factors involved in this event under physiological conditions.

After GVBD, mitochondria continue to aggregate around the perinuclear area at $\mathrm{Ml}$ and anaphase I, which is probably related to the high energy requirement of meiotic events, such as spindle assembly, chromatin condensation and movement, and polar body emission. In laboratory mice, perinuclear accumulation of mitochondria is one aspect of the developmental programme of cytoplasmic maturation observed between GVBD and MI (Van Blerkom and Runner, 1984; Van Blerkom, 1991; Calarco, 1995). The strong staining of mitochondria in the perinuclear area may be caused by either mitochondrial translocation or by activation of mitochondria in situ. Although examination of bovine oocytes demonstrates that the subcellular organization of the oocyte cytoplasm remains unchanged during maturation to MII (Van Blerkom et al., 1990), it has been reported that mitochondria actively relocate during oocyte maturation and fertilization in several species (Bavister and Squirrell, 2000). The present study revealed no changes in active mitochondrial distribution of pig oocytes treated with $100 \mu \mathrm{mol}$ nocodazole $\mathrm{I}^{-1}$, a concentration that inhibits microtubule assembly (Sun et al., 2001), but aggregation of mitochondria was found in oocytes treated with $500 \mathrm{nmol}$ cytochalasin $\mathrm{B} \mathrm{I}^{-1}$, a concentration that blocks microfilament assembly (Sun et al., 2001). In mouse oocytes, mitochondrial movement toward the GV area is mediated by microtubules but not by microfilaments (Van Blerkom and Bell, 1986). There is a

the cortex of GV oocytes derived from small follicles. $(\mathrm{d}, \mathrm{e}, \mathrm{f})$. Examples of progressive inward translocation and aggregation of mitochondria around the GV in oocytes derived from large follicles. Arrows indicate the accumulation of mitochondria in the vicinity of the GVs. (g) Mitochondria accumulated in the perinuclear region (arrow) after $30 \mathrm{~h}$ of culture. ( $\mathrm{h}$ ) Mitochondria were translocated to the inner cytoplasm, but only a few were found in the centre of the cytoplasm in mature oocytes after $44 \mathrm{~h}$ of culture. Note strong mitochondrial staining in the first polar body (arrow). (i) An observation plane through the cortex of a mature oocyte, showing homogeneous distribution of larger mitochondrial foci. Arrow indicates the first polar body. (j) An in vivo matured oocyte, showing the distribution of mitochondria throughout the cytoplasm. (k) An example showing the absence of mitochondria in the central cytoplasm of an in vivo matured oocyte. (l) An in vivo matured oocyte, showing the absence of mitochondria in the peripheral cytoplasm. Note the strong staining of the polar body (arrow). Scale bar represents $40 \mu \mathrm{m}$. 

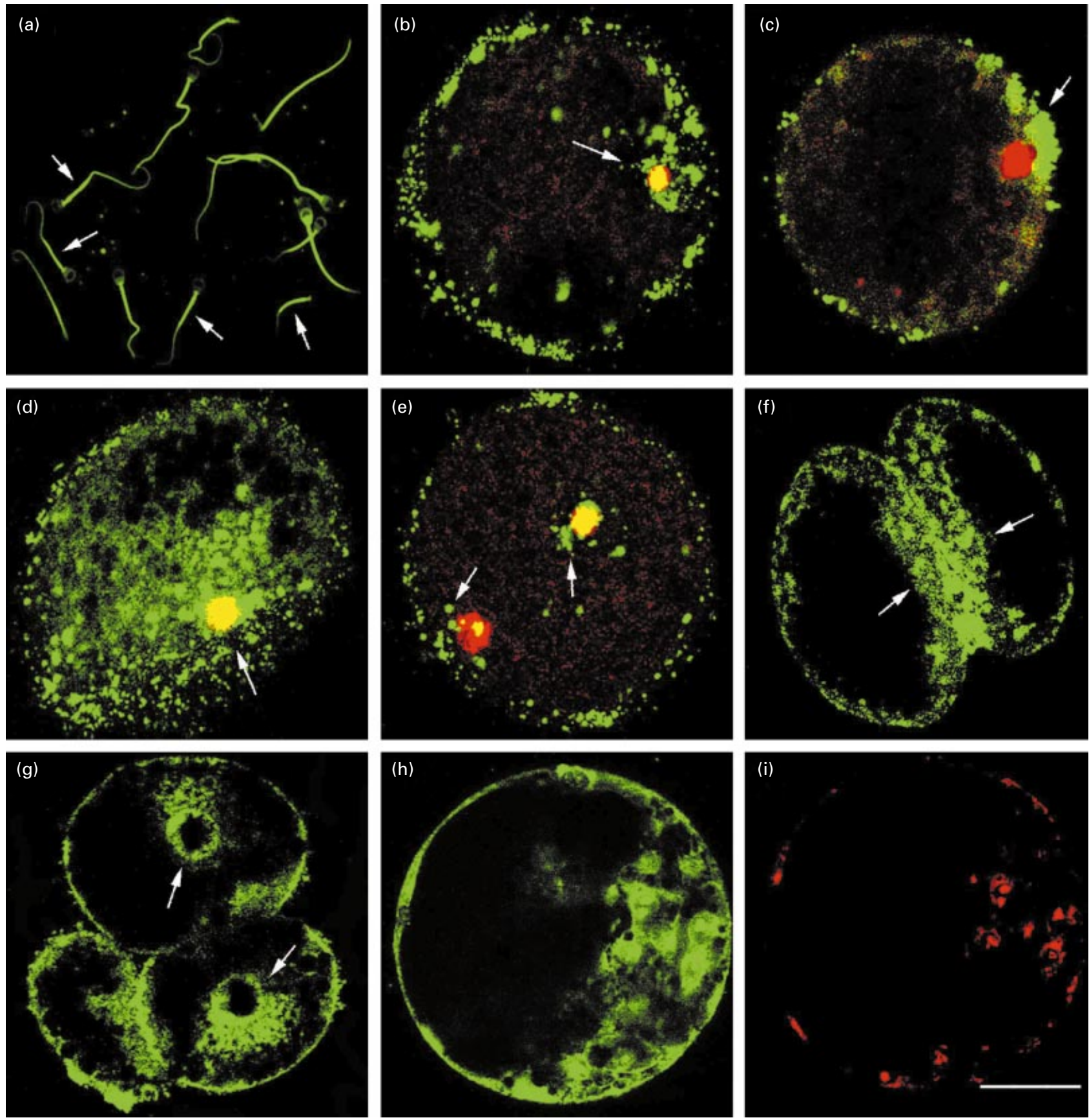

Fig. 2. Mitochondrial distribution in fertilized eggs and early embryos in pigs. (a) The tails, especially the principal segments (arrows), of spermatozoa that attached to the zona surface of fertilized eggs were stained green. (b) A fertilized egg, indicating the mitochondria distributed close to the plasma membrane and in the vicinity of a pronucleus (arrow). (c) Mitochondrial aggregation between the pronucleus and the plasma membrane (arrow). (d) In addition to the perinuclear distribution (arrow), some mitochondrial foci were observed in the inner cytoplasm of a few fertilized eggs. (e) Mitochondrial aggregation around both pronuclei (arrows). (f) Mitochondrial aggregation in the region in which the blastomeres are still in contact (arrows) during early cleavage. (g) Mitochondrial clustering around the blastomere nuclei (arrows) and in the cortex of the cell. (h,i) Mitochondria present in both inner cell mass cells and trophectoderm cells of blastocysts. Green, mitochondria; red, chromatin; yellow, overlapping of green and red. Scale bar represents $40 \mu \mathrm{m}$.

temporal, spatial and developmental relationship between the location of microtubule-organizing centres and the progressive translocation of mitochondria to the nuclear region (Van Blerkom, 1991). This finding indicates that strong staining of mitochondria in the perinuclear area in pig oocytes is caused by active mitochondrial translocation and that, as in mouse oocytes, mitochondrial translocation is mediated by microtubules but not by microfilaments. 

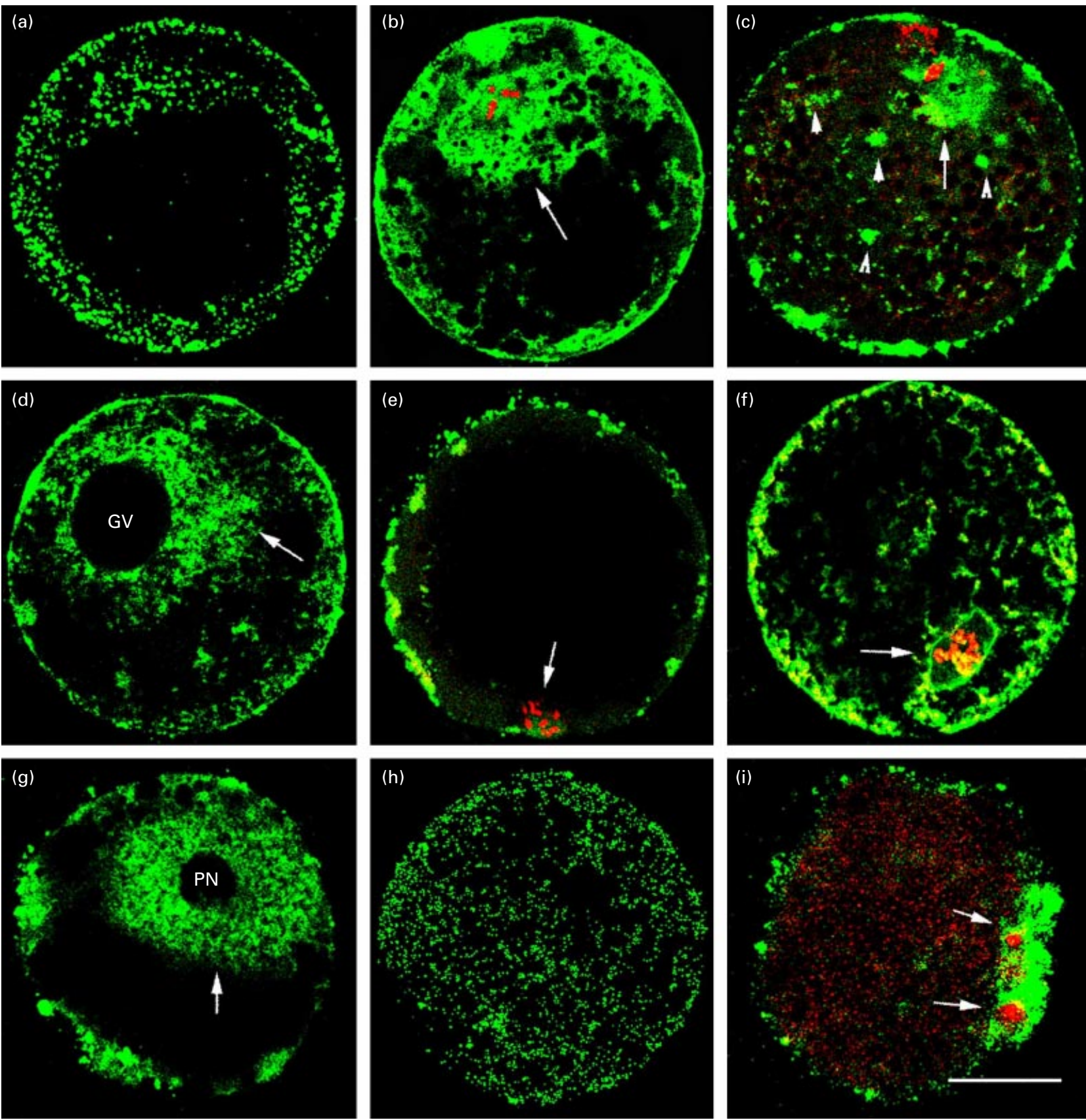

Fig. 3. Regulation of mitochondrial translocation by microtubule and microfilament modulators during maturation and fertilization of pig oocytes. (a) Mitochondrial distribution in a control oocyte after $44 \mathrm{~h}$ of culture. (b) Mitochondrial accumulation in the vicinity (arrow) of the metaphase I (MI) chromosomes in an oocyte arrested at MI by cytochalasin B after $44 \mathrm{~h}$ of culture. (c) Mitochondrial foci (arrowheads) distributed in the cytoplasm, in addition to the accumulation of mitochondria around the MI chromosomes (arrow). (d) Mitochondrial distribution around the GV (arrow) in an oocyte arrested at the GV stage treated with cytochalasin B after $44 \mathrm{~h}$ of culture. (e) Inhibition of inward movement of mitochondria toward the nuclear area (arrow) in an oocyte treated with nocodazole for $44 \mathrm{~h}$. (f) A few mitochondria are distributed in the vicinity of Ml chromosomes (arrow) in some oocytes treated with nocodazole for $44 \mathrm{~h}$. (g) Aggregation of mitochondria around the pronucleus (arrow) in a fertilized egg treated with cytochalasin B. (h) Distribution of mitochondria in a fertilized egg after microtubule disruption with nocodazole. (i) Inhibition of centration of pronuclei and accumulation of mitochondria around the pronuclei (arrows) in a fertilized egg treated with taxol. Green, mitochondria; red, chromatin; yellow, overlapping of green and red. Scale bar represents $40 \mu \mathrm{m}$. 
Observations in the present study revealed that larger but scarcely distributed mitochondrial foci formed after in vitro maturation of pig oocytes. This observation is consistent with electron microscopy. Cran (1985) reported a decrease in the number of mitochondria such that at $50 \mathrm{~h}$ after hCG the number of mitochondria was only one-third of that at 0 h. Concomitantly, there was $>300 \%$ increase in individual mitochondrial volume. In addition, small groups of mitochondria became enveloped in cisternae of endoplasmic reticulum. Thus, the larger mitochondrial foci in the mature oocytes in the present study, as revealed by MitoTracker staining, may represent the increase in mitochondrial volume and the aggregation of mitochondria.

Peripheral mitochondria showed an even spatial distribution during in vitro maturation of bovine oocytes (Hyttel et al., 1986) and in vivo maturation of mouse and hamster oocytes (Tokura et al., 1993; Barnett et al., 1996). However, Calarco (1995) reported that fully mature mouse oocytes showed obvious polarity of mitochondrial distribution. The present study revealed that accumulation of mitochondria around the MII spindle did not occur, but instead they were distributed homogeneously in the ooplasm except for in the inner cytoplasm during in vitro maturation of pig oocytes. The absence of mitochondria in the inner cytoplasm may be caused by in vitro culture conditions and represents poor cytoplasmic maturation of in vitro matured oocytes, as both electron microscopy (Cran, 1985) and MitoTracker staining (present study) show that mitochondria are dispersed to the inner cytoplasm in most oocytes matured in vivo.

Staining of active mitochondria was strong in the first polar bodies of both in vivo and in vitro matured oocytes. Intensive mitochondrial staining was also observed in the second polar body of fertilized hamster (Barnett et al., 1996) and pig eggs (present study). Mitochondria are the most apparent cytoplasmic organelle in the first polar body of rabbit oocytes matured in vivo as revealed by electron microscopy (Zamboni and Mastroianni, 1966).

There is little information regarding mitochondrial changes during oocyte fertilization and early embryo development in pigs. The present study showed that, in fertilized eggs, the homogeneous distribution of mitochondria in mature oocytes was no longer apparent and that mitochondria aggregated to the peri-pronuclear region. This finding is consistent with results obtained in mouse, hamster and human zygotes, in which mitochondria showed perinuclear accumulation (Tokura et al., 1993; Barnett et al., 1996; Sun et al., 1996; Van Blerkom et al., 2000). The accumulation of mitochondria in the perinuclear area indicates that pronuclear apposition and syngamy may require high concentrations of ATP or free calcium.

The present study also revealed that aggregation of mitochondria in the pronuclear area was mediated by microtubules: whereas exposure of sperm-penetrated pig oocytes to taxol, which promotes the assembly of microtubules, did not affect mitochondrial aggregation in the vicinity of pronuclei, the centration of pronuclei was inhibited. When mouse oocytes at the GV or GVBD stages were treated with taxol, bundles of microtubules formed and large aggregates of mitochondria were arranged along the microtubule bundles at the periphery of the nucleus (Rime, 1987).

As reported in hamsters (Barnett et al., 1996), the consistency of perinuclear clustering and cortical ring of mitochondria in the blastomeres was the most marked feature of cleavage of pig early embryos. In addition, more mitochondria were located in the region between the blastomeres. The translocation of active mitochondria was correlated with the ability of mouse embryos to develop in vitro. In mouse embryos with a developmental block, the translocation of mitochondria was inhibited, whereas translocation of mitochondria was restored when the developmental block was released by adding superoxide dismutase or thioredoxin to the culture medium (Tokura et al., 1993). In contrast to hamster blastocysts, in which mitochondria were detected most readily in trophectoderm cells (Barnett et al., 1996), mitochondria were distributed homogeneously in both inner cell mass cells and trophoctoderm cells in pig blastocysts.

In conclusion, the present study revealed several new characteristics of mitochondrial distribution during pig oocyte maturation: (i) mitochondria accumulate in the perinuclear area from the GV stage to anaphase I; (ii) fewer but larger mitochondrial foci form and relocate to the inner cytoplasm in mature oocytes, and mitochondrial translocation in oocytes matured in vitro is not as complete as in oocytes matured in vivo; and (iii) strong staining of active mitochondria is observed in the first polar body. Mitochondria aggregate to the pronuclear area in fertilized eggs, and perinuclear clustering of mitochondria is also a marked feature in early cleavage. Mitochondria are distributed homogeneously in both inner cell mass cells and trophectoderm cells of blastocysts. Finally, the apparent translocation of mitochondria is mediated by microtubules but not by microfilaments in pig oocytes.

This study was supported by grants from the University of Missouri-Columbia to H. Schatten, Food for the 21st Century Program to R. S. Pranther. Q. Y. Sun was supported by the 973 project (G1999055902) and Knowledge Innovation Program while working at $\mathrm{H}$. Schatten's laboratory as a research associate. This manuscript is a contribution from the Missouri Agricultural Experimental station: journal series number 13090.

\section{References}

Abeydeera LR, Wang WH, Prather RS and Day BN (1998) Maturation in vitro of pig oocytes in protein-free media: fertilisation and subsequent development in vitro. Biology of Reproduction 58 1316-1320

Barnett DK, Kimura J and Bavister BD (1996) Translocation of active mitochondria during hamster preimplantation embryo development studied by confocal laser scanning microscopy Developmental Dynamics 205 64-72

Barnett DK, Claton MK, Kimura J and Bavister BD (1997) Glucose and phosphate toxicity in hamster preimplantation embryos involves disruption of cellular organization, including disruption of active mitochondria Molecular Reproduction and Development 48 227-237 
Bavister BD (2000) Interactions between embryos and the culture media Theriogenology 53 619-626

Bavister BD and Squirrell JM (2000) Mitochondrial distribution and function in oocytes and embryos Human Reproduction Supplement 2 189-198

Calarco PG (1995) Polarization of mitochondria in the unfertilised mouse oocytes Development and Genetics 16 36-46

Cran DG (1985) Qualitative and quantitative structural changes during pig oocyte maturation Journal of Reproduction and Fertility 74 237-245

Cran DG, Moor RM and Hay MF (1980) Fine structure of the sheep oocytes during antral follicle development Journal of Reproduction and Fertility 59 125-132

Downs SM and Mastropolo AM (1994) The participation of energy substrates in the control of meiotic maturation in murine oocytes Developmental Biology 162 154-168

Eppig JJ (1982) The relationship between cumulus cell-oocyte coupling, oocyte meiotic maturation and cumulus expansion Developmental Biology 89 268-272

Fleming WN and Saacke RG (1972) Fine structure of the bovine oocyte from mature Graaffian follicles Journal of Reproduction and Fertility 29 203-213

Han YM, Wang WH, Abeydeera LR, Petersen AL, Kim JH, Murphy D, Day BN and Prather RS (1999) Pronuclear localization before the first cell division determines ploidy of polyspermic pig embryos Biology of Reproduction 61 1340-1346

Hyttel P, Xu KP, Smith S and Greve T (1986) Ultrastructure of in vitro oocyte maturation in cattle Journal of Reproduction and Fertility 78 615-625

Kaufman ML and Homa ST (1993) Defining a role for calcium in the resumption and progression in the pig oocytes Journal of Experimental Zoology 265 69-76

Lane M and Bavister BD (1998) Calcium homeostasis in early hamster preimplantation embryos Biology of Reproduction 59 1000-1007

Petters RM and Wells KD (1993) Culture of pig embryos Journal of Reproduction and Fertility Supplement 48 61-73

Prather RS (1993) Nuclear control of early embryonic development in domestic pigs Journal of Reproduction and Fertility Supplement $\mathbf{4 8}$ 17-29

Prather RS and Day BN (1998) Practical considerations for the in vitro production of pig embryos Theriogenology 49 23-32

Rime H, Jessus C and Ozon R (1987) Distribution of microtubules during the first meiotic cell division on the mouse oocyte: effect of taxol Gamete Research 17 1-13

Sun QY and Qin PC (1989) Ultrastructure of bovine oocyte development Journal of Shandong Agricultural University (in Chinese) 4 9-14

Sun QY and Qin PC (1990) Nuclear changes during bovine oocyte development Journal of Northeast Agricultural College (in Chinese) 21 234-238
Sun QY, Gao SR and Chen DY (1996) Chronological and morphological progression of the nucleus during mouse oocyte maturation and fertilisation in vitro. Developmental and Reproductive Biology 5 24-33

Sun QY, Lai L, Park KW, Kühholzer B, Prather RS and Schatten H (2001) Dynamic events are differently regulated by microfilaments, microtubules, and mitogen-activated protein kinase during porcine oocyte maturation and fertilization in vitro. Biology of Reproduction 64 879-889

Trimarchi JR, Liu L, Porterfiled DM, Smith PJ and Keefe DL (2000) Oxidative phosphorylation-dependent and -independent oxygen consumption by individual preimplantation mouse embryos Biology of Reproduction 62 1866-1874

Tokura T, Noda Y, Goto Y and Mori T (1993) Sequential observation of mitochondrial distribution in mouse oocytes and embryos Journal of Assisted Reproduction and Genetics 10 417-426

Van Blerkom J (1991) Microtubule mediation of cytoplasmic and nuclear maturation during the early stages of resumed meiosis in cultured mouse oocytes Proceedings National Academy of Sciences USA 88 5031-5035

Van Blerkom J and Bell H (1986) Regulation of development in the fullygrown mouse oocyte: chromosome-mediated temporal and spatial differentiation of the cytoplasm and plasma membrane Journal of Embryology and Experimental Morphology 93 213-238

Van Blerkom J and Runner MN (1984) Mitochondrial reorganization during resumption of arrested meiosis in the mouse oocyte American Journal of Anatomy 171 335-355

Van Blerkom J, Bell H and Weipz D (1990) Cellular and developmenta biological aspects of bovine meiotic maturation, fertilization and preimplantation embryogenesis in vitro. Journal of Electron Microscopy Techniques 16 298-332

Van Blerkom J, Davis P and Lee J (1995) ATP content of human oocytes and developmental potential and outcome after in vitro fertilization and embryo transfer Human Reproduction 10 415-424

Van Blerkom J, Davis P and Alexander S (2000) Differential mitochondrial distribution in human pronuclear embryos leads to disproportionate inheritance between blastomeres: relationship to microtubular organization, ATP content and competence Human Reproduction 15 $2621-2633$

Zamboni L and Mastroianni J, Jr (1966) Electron microscopic studies on rabbit ova: I. The follicular oocytes Journal of Ultrastructure Research $\mathbf{1 4}$ 95-117

Received 8 November 2000.

First decision 16 January 2001.

Accepted 27 March 2001. 
